\title{
Internal Demagnetizing Factor in Ferrous Metals
}

\author{
Jenő Takács, ${ }^{1}$ György Kovács, ${ }^{2}$ and Lajos K. Varga ${ }^{3}$ \\ ${ }^{1}$ Department of Engineering Science, University of Oxford, Parks Road, Oxford OX1 3PJ, UK \\ ${ }^{2}$ Department of Materials, Physics of Eotvos Lorand University, P.O. Box 32, Budapest 1518, Hungary \\ ${ }^{3}$ Research Institute for Solid State Physics and Optics, P.O. Box 49, Budapest 1525, Hungary \\ Correspondence should be addressed to Jenő Takács, jenotakacs@aol.com
}

Received 26 July 2012; Accepted 19 September 2012

Academic Editor: Chih-Ming Chen

Copyright ( $\odot 2012$ Jenő Takács et al. This is an open access article distributed under the Creative Commons Attribution License, which permits unrestricted use, distribution, and reproduction in any medium, provided the original work is properly cited.

\begin{abstract}
Modelling the saturation major loop of a ferrous metal produces the intrinsic magnetization parameters; fitting the measured commutation curve, however, can yield different results. The relation of the intrinsic loci of the vertices of the minor loops $\left(N_{D}=0\right)$ to the experimental curve $\left(N_{D} \neq 0\right)$ is investigated. The two-way transformation between the two curves is formulated in closed mathematical form with the help of the internal demagnetization factor, $N_{D}$. The method is applied to four ferrous metals, with widely different intrinsic properties (soft nonoriented Fe-Si steel, normalized low carbon steel, and Finemet in nanocrystalline and amorphous state) supporting the predictions of the proposal. The developed relationship is model independent and it is shown that the $N_{D}$ factor depends linearly on coercivity based on experimental evidence.
\end{abstract}

\section{Introduction}

A large number of the measurements of ferrous substances are aimed at finding the intrinsic material properties [1] of the tested ferrous sample (as defined by Fiorillo). Due to the ever-presence of demagnetization field, various measuring methods have been developed to minimize its effect. The most commonly accepted way is to make the sample turn into a closed magnetic circuit, such as a toroid or an Epstein square [1-3]. Although these two methods are not completely free from the ever-present internal demagnetization, they suffer the least from it [1]. Researchers went into great length to include the internal demagnetization force into current models like Preisach, Jiles, Stoner-Wohlfarth [2, 4, 5], and so forth, leading to complicated, so called, dynamic versions.

The saturated major hysteresis loop of the sample carries all the intrinsic magnetic parameters directly recoverable from the measured data. Within this loop lie the unhysteretic loci of the vertices of the symmetrical minor loops, the only curve, which belongs to both the ascending and descending branches of the hysteresis loops $[5,6]$.

A proposal is put forward in this paper to show the relationship between the intrinsic curve and the loci of vertices of the measured minor loops. This relationship between the two curves, independent of models, is formulated in closed mathematical form and its prediction is verified by the experimental data obtained from four different ferrous samples.

Once the intrinsic locus $\left(M_{01}\right.$ for $\left.N_{d}=0\right)$ is modelled from saturation or minor loop data, by using any of the static models, the measured curve $\left(N_{d} \neq 0\right)$ can be calculated from the proposed formulation below with optimization of the $N_{d}$ parameter value as specified by Jiles [2] and used by Fiorillo [1].

\section{The Intrinsic Loci of Vertices}

The intrinsic commutation curve $\left(N_{D}=0\right)$ is the locus of the return points or the maxima of the set of symmetrical minor loops. It is a single-valued function and in spite of having no hysteresis, it carries all the hysteretic properties of the ferrous material obtained from the saturation data [7].

$M_{0}$ is the arithmetic mean of the ascending and descending magnetization functions, $M_{u}$ and $M_{d}$, with equal application to $N_{D}=0$ case as well.

$$
M_{0}=\frac{\left(M_{u}+M_{d}\right)}{2} .
$$


Due to the difference between $M_{0}$ and the measured commutation curve, hysteresis loop modelling based solely on the measured commutation curve can produce different parameters, when no molecular interaction is assumed $[6,8$, 9].

\section{The Effective Field and Its Implications}

For the description of the effect of the internal demagnetizing field we will use the concept of the effective field, which is analogous to the Weiss mean field as defined and used by other authors [2, 10, 11]. Although in simple cases, proportionality is assumed between $\mathbf{H}_{\mathbf{D}}$ interaction field and $\mathbf{M}$ magnetization vector, this can only be regarded as a linear approximation $[12,13]$ expressed as $H_{D}=N_{D} M$ in scalar form, leading to

$$
H_{\mathrm{eff}}=H+N_{D} M
$$

where $N_{D}$ is the internal demagnetization factor.

For simplicity we will use normalised quantities in further calculations, where the lower case letters will represent the normalised quantities of the physical equivalents, denoted by the same capital letters.

$m_{0}$ denote the normalised $M_{0}$ function in (1). With the normalized effective field, $h_{\text {eff }}, m_{0}$ can be expressed as:

$$
m_{0}=f\left(h_{\mathrm{eff}}\right)=f\left(h+n_{D} m_{0}\right)
$$

where $n_{D}$ is also normalized. ( 1 Tesla $=8 \cdot 10^{5} \mathrm{~A} / \mathrm{m}$. The internal demagnetization factor has unity dimension only when the magnetization is measured in $\mathrm{A} / \mathrm{m}$.).

The first derivative of $m_{0}$ by $h$ in (3) leads to an expression, which shows a character similar to the feedback in an electrical circuit [14].

This expression describes a well-known relationship between the inherent $\left(\mu_{i}\right)$ and the effective $\left(\mu_{\text {eff }}\right)$ permeabilities $[1,2]$.

For most magnetic substances the value of $N_{D}$ is small in the order of $\sim-10^{-5}$ with unity dimension [1]. Consider

$$
\frac{d m_{0}}{d h}=\frac{d m_{0} / d h_{\mathrm{eff}}}{1-n_{D}\left(d m_{0} / d h_{\mathrm{eff}}\right)} .
$$

By using the hysteretic model [6] (see also the appendix), the integral of (4) by $h$ leads us to the following expression for $m_{0}$, when

$$
\begin{aligned}
\frac{d m_{0}}{d h}=\sum_{k=1}^{n} \frac{a_{k}}{2}[ & \frac{\sec h^{2}\left(h_{k u \text { eff }}\right)}{1-n_{D} a_{k} \sec h^{2}\left(h_{k u \text { eff }}\right)} \\
& \left.+\frac{\sec h^{2}\left(h_{k d \text { eff }}\right)}{1-n_{D} a_{k} \sec h^{2}\left(h_{k d \text { eff }}\right)}\right] .
\end{aligned}
$$

After integration:

$$
\begin{aligned}
m_{0}=\sum_{k=1}^{n} \frac{a_{k}}{2}\left\{-\sqrt{a_{k}}\right. & \frac{\operatorname{Arctanh}\left[\left(\sqrt{a_{k} n_{D}} \tanh \left[\alpha_{k}\left(h-h_{c k}\right)\right] / \sqrt{-1+a_{k} n_{D}}\right)\right]}{\sqrt{n_{D}\left(-1+a_{k} n_{D}\right)}} \\
& \left.-\sqrt{a_{k}} \frac{\operatorname{Arctanh}\left[\left(\sqrt{a_{k} n_{D}} \tanh \left[\alpha_{k}\left(h+h_{c k}\right)\right] / \sqrt{-1+a_{k} n_{D}}\right)\right]}{\sqrt{n_{D}\left(-1+a_{k} n_{D}\right)}}\right\} .
\end{aligned}
$$

When the integration constants are appropriately chosen, this form of $m_{0}$ is equivalent to the one given in (A.5).

The intrinsic locus is entirely a theoretical concept. It was introduced for the free (Gibbs) energy calculations [2]. It assumes zero internal demagnetization in a system, where a $m$ moment can freely move around under the influence of $H$ external excitation field without any hindrance from the interaction between the magnetic moments.

We must remind the reader that, for various ferromagnetic substances, this internal demagnetization constant $N_{D}$ is given traditionally in a numerical value with unity dimension (i.e., when both the $H$ and $B$ measured in $\mathrm{A} / \mathrm{m}$ ). When different unitary system is used, $N_{D}$ has a different physical dimension and must be normalised (see $n_{D}$ as normalised $N_{D}$ ).

\section{Experimental Verification}

To verify the predictions of the proposed method, it was applied initially to two ferrous materials with very different characters. The first was a soft steel NO Fe-Si with $67.5 \mathrm{~A} / \mathrm{m}$ coercivity [15], shown in Figure 1. The second material was a normalized low carbon steel (AISI 1040) with coercivity of $450 \mathrm{~A} / \mathrm{m}$ (see Figure 5). Following the excellent results, the same experiment was also repeated later on two other samples; Finemet in nanocrystalline and amorphous (as cast) state. The detailed data of those samples are not included in this paper due to its limited size. All measurements were carried out under identical conditions by using triangular excitation of $f=0.001 \mathrm{~Hz}$ on toroid samples with geometrical details as follows: $D_{e}=25 \mathrm{~mm}, D_{\text {int }}=15 \mathrm{~mm}$ and thickness $d=0.5 \mathrm{~mm}$ [1]. In making the identical toroid samples, extreme care was taken to avoid any changes in magnetic properties due to mechanical handling of the materials.

For numerical calculations we used the hyperbolic model for its simplicity and speed and the Mathematica program interactively. A brief summary of the model is given in the appendix. For further details we refer the reader to the literature $[6,16,17]$. 


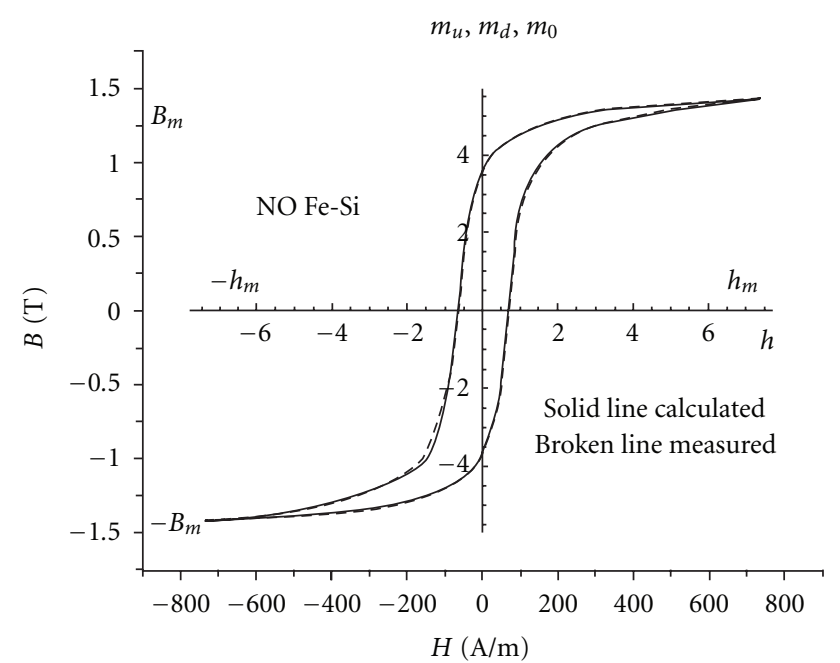

Figure 1: The measured and the modelled major loop of NO Fe-Si soft steel.

4.1. NO Fe-Si. Starting with conveniently chosen parameter values at the beginning with subsequent changes of the parameters, new curves are calculated and compared with the measured one.

When the iteration produced the best fit to the measured curve, the normalized and the equivalent physical values can be easily read from the two coordinate systems (normalization) as shown in Figures 1, 2, and 3.

The first sample (see Figure 1) was modelled with the following normalized and physical parameters:

$$
\begin{aligned}
& a_{1}=3.18, a_{2}=1.45, a_{3}=1.09, \\
& \alpha_{1}=2.75, \alpha_{2}=0.56, \alpha_{3}=0.134, \\
& h_{c 1}=0.675, h_{2 c}=0.57, h_{c 3}=2.2, \\
& h_{m}=7.35 \text { equivalent to: } \\
& A_{1}=0.842 \mathrm{~T}, A_{2}=0.384 \mathrm{~T}, A_{3}=0.288 \mathrm{~T} . \\
& H_{c 1}=67.5 \mathrm{~A} / \mathrm{m}, H_{c 2}=57 \mathrm{~A} / \mathrm{m}, H_{c 3}=220 \mathrm{~A} / \mathrm{m}, H_{m}= \\
& 735 \mathrm{~A} / \mathrm{m} . \\
& \text { with normalization of } 1 \mathrm{~h}=H 100 \mathrm{~A} / \mathrm{m} \text { and } 1 \mathrm{~m}= \\
& M 0.2647 \mathrm{~T} \text {. }
\end{aligned}
$$

The measured and the modelled $m_{0}$ curves for $n_{D}=$ $-0.151\left(N_{D}=-57 \mathrm{~A} / \mathrm{Tm}\right.$, or $\left.-7 \cdot 10^{-5}\right) . n_{D}=0$ are depicted in Figure 2. For the symbols see the appendix.

In order to check the accuracy of the transformation, between the two curves, 25 of the minor loops were measured with maximum field excitation values between $h_{m}=5.01$, $H m=501 \mathrm{~A} / \mathrm{m}$ and $h_{m}=0.152, H m=15.2 \mathrm{~A} / \mathrm{m}$. For all the minor loops measured, the corresponding loops were calculated for the reduced maximum magnetization at $n_{D}=$ 0 and $n_{D}=-0.151$.

For clarity, only one of the minor loops is shown in Figure 3 for the peek excitation field value of $111.3 \mathrm{~A} / \mathrm{m}$.

All the calculated loops had an excellent fit to the equivalent measured loops for the same $N_{D}$ value. The proposed method is applicable to all measured hysteretic data, where the external demagnetization field is eliminated or reduced to a negligible level.

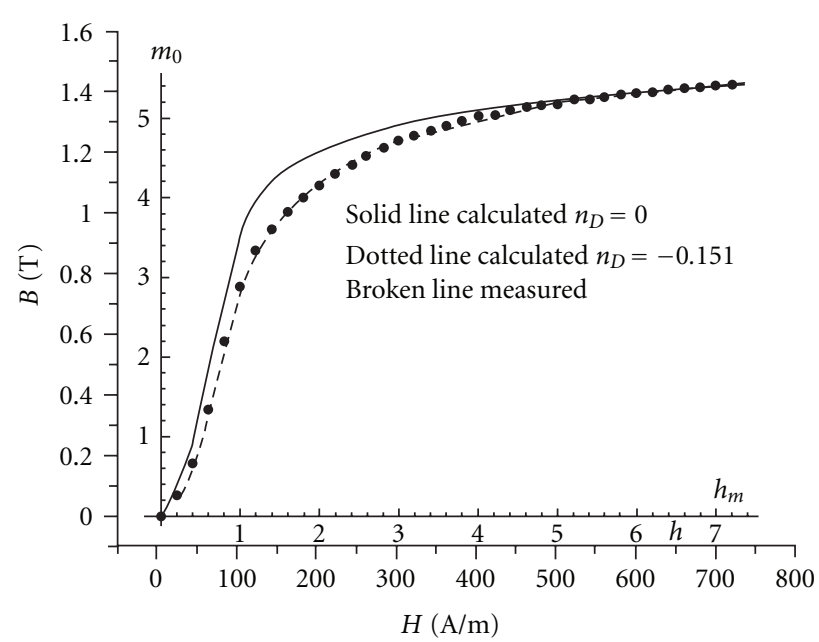

Figure 2: Measured (broken line), modelled (dotted line), and intrinsic (solid line) commutation curves.

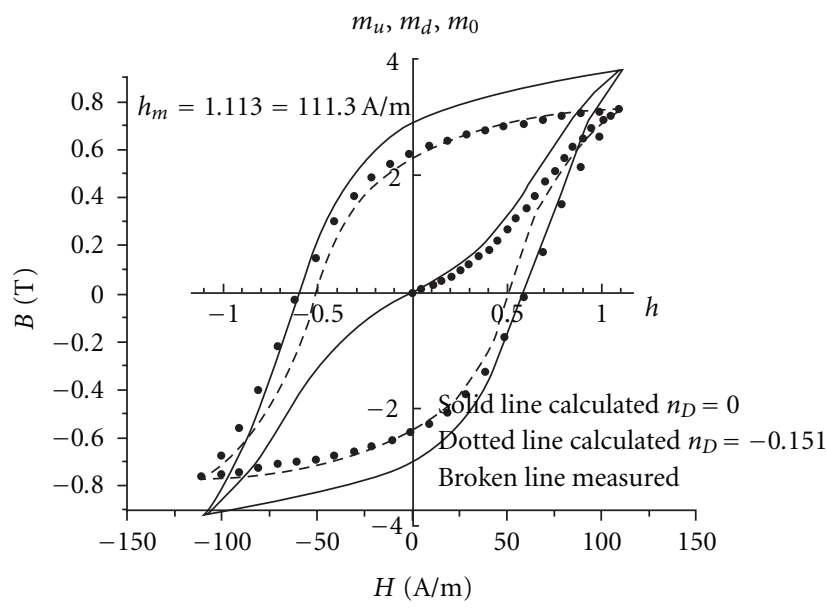

FIgURE 3: Calculated NO Fe-Si minor hysteresis loops for $n_{D}=0$ (solid line) and $n_{D}=-0.151$ (dotted line), fitted to the measured loop (broken line) with internal demagnetization.

4.2. Low Carbon Steel Toroid (AISI 1040). For the second sample, we selected a toroid from low carbon steel with coercivity of $450 \mathrm{~A} / \mathrm{m}$. The use of the same experimental setup has yielded the following parameters in normalized and physical units:

$$
\begin{aligned}
& a_{1}=2.68, a_{2}=2.08, a_{3}=1.6, \\
& \alpha_{1}=3.52, \alpha_{2}=0.47, \alpha_{3}=0.09, \\
& h_{c 1}=0.45, h_{c 2}=0.44, h_{c 3}=0.9 \text { and } h_{m}=7.35,
\end{aligned}
$$

equivalent to:

$$
\begin{aligned}
& A_{1}=0.64 \mathrm{~T}, A_{2}=0.5 \mathrm{~T}, A_{3}=0.38 \mathrm{~T}, \\
& H_{c 1}=450 \mathrm{~A} / \mathrm{m}, H_{c 2}=440 \mathrm{~A} / \mathrm{m}, H_{c 3}=900 \mathrm{~A} / \mathrm{m}, \text { and } \\
& H_{m}=7350 \mathrm{~A} / \mathrm{m},
\end{aligned}
$$

with normalization of $1 \mathrm{~h}=H 1000 \mathrm{~A} / \mathrm{m}$ and $1 \mathrm{~m}=$ M0.24 T.

The measured and the modelled hysteresis loops are depicted in Figure 4 with the intrinsic and the measured 


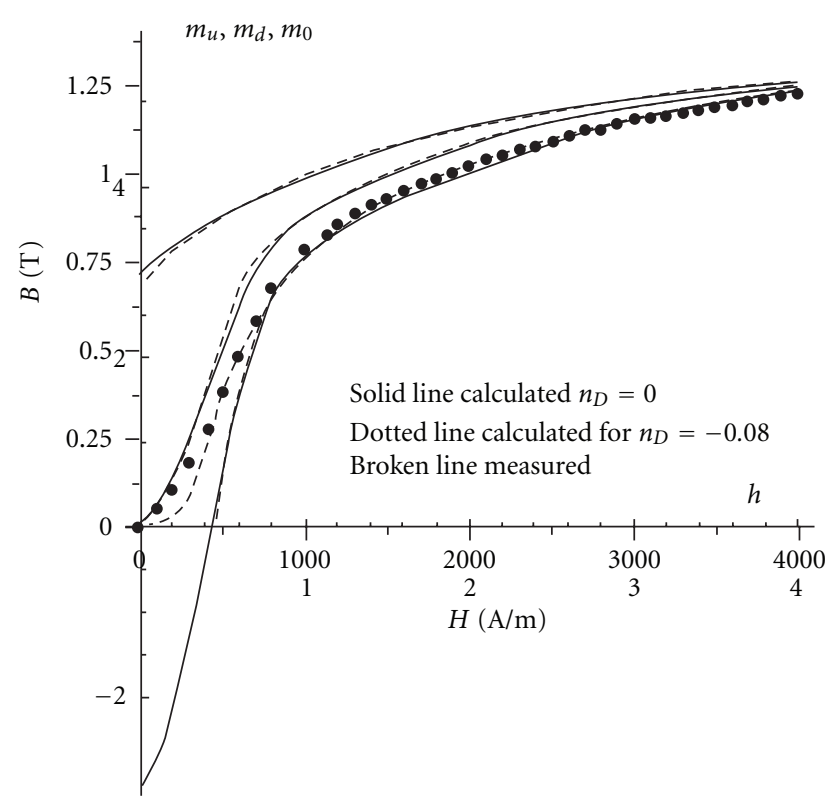

Figure 4: Measured (broken line) and modelled (solid line) hysteresis and the commutation curves for $n_{D}=0$ and $n_{D}=-0.08$ (dotted line) in the first quadrant for the low carbon steel (AISI 1040).

commutation curves. The curves are shown in the first quadrant only for better visual resolution.

Following the calculation of the intrinsic parameters, the measured commutation curve was modelled with $n_{D}=$ $-0.08\left(N_{D}=-333.3 \mathrm{~A} / \mathrm{Tm}\right.$ or $\left.-4.1 .10^{-4}\right)$, which yielded the best result, giving an excellent fit to the measured curve.

\section{5. $N_{d}$ as a Function of Coercivity}

The experimental results have indicated that $N_{D}$ is greatly dependent on the coercivity of the sample. To verify this dependency, four samples, listed under Section 4, were tested for this purpose, with coercivity $H_{c}$ ranging between $1.6 \mathrm{~A} / \mathrm{m}$ and $450 \mathrm{~A} / \mathrm{m}$. Figure 5 depicts the relationship between $N_{D}$ and $H_{c}$.

The graph, in Figure 5, was constructed by using the parameters of the samples listed below, whose coercivity values change in steps approximately by an order of magnitude:

Finemet: $H_{c}=1.6 \mathrm{~A} / \mathrm{m}, N_{D}=-0.729 \mathrm{~A} / \mathrm{Tm}$ or $-0.9 \cdot 10^{-6}$,

Finemet in amorphous state: $H_{c}=12.6 \mathrm{~A} / \mathrm{m}, N_{D}=$ $-4.96 \mathrm{~A} / \mathrm{Tm}$ or $-6.2 \cdot 10^{-6}$,

NO Fe-Si: $H_{c}=67.5 \mathrm{~A} / \mathrm{m}, N_{D}=-57 \mathrm{~A} / \mathrm{Tm}$ or $-7 \cdot 10^{-5}$,

Low carbon steel (AISI 1040): $H_{c}=450 \mathrm{~A} / \mathrm{m}, N_{D}=$ $-333 \mathrm{~A} / \mathrm{Tm}$ or $-4 \cdot 16.10^{-4}$.

The graph shows $N_{D}$ linear dependency on $H_{c}$ coercivity and based on experimental evidence, it can be approximated as

$$
N_{D} \approx \mu_{0} \frac{H_{c}}{B_{m}},
$$

where $B_{m}$ represents the saturation magnetization.

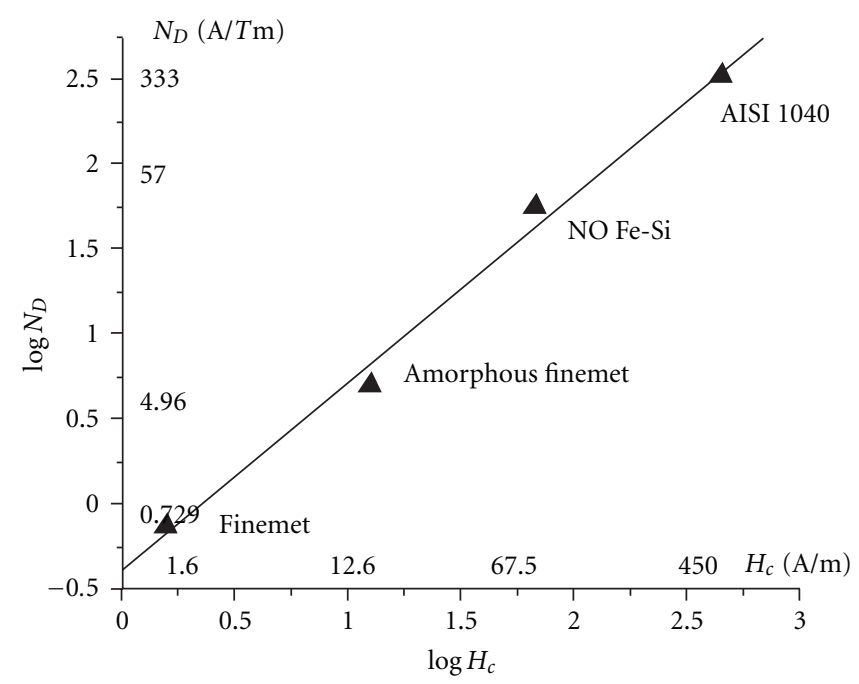

FIgURe 5: $N_{D}$ as the function of coercivity $H_{c}$.

\section{Conclusions}

The relation between the intrinsic $\left(N_{D}=0\right)$ and the measured $\left(N_{D} \neq 0\right)$ loci of the vertices of the minor loops was investigated. By using the effective field, this relationship was formulated. The method was subjected to tests on four magnetic materials with widely ranging magnetic properties. The test results show that the mathematical approach, presented here, describes the relation well. The $N_{D}$ factor linear dependence on coercivity, based on empirical evidence, was also demonstrated. The paper shows that magnetic parameters $\left(H_{c}\right.$ and $\left.B_{m}\right) N_{D}$ can be estimated very close to the real value.

The proposal's aim is to recover the intrinsic magnetization properties from the measured commutation curves.

\section{Appendix}

The characteristic equations of the hyperbolic model in canonic form:

$$
\begin{gathered}
m_{u}=\sum_{k=1}^{n}\left[a_{k} f_{u k}+f_{0 k}\left(h_{m}\right)\right], \\
m_{d}=\sum_{k=1}^{n}\left[a_{k} f_{d k}-f_{0 k}\left(h_{m}\right)\right], \\
f_{u k, d k}=\tanh \left[\alpha_{k}\left(h \mp h_{c k}\right)\right], \\
f_{0 k}=\sum_{k} \frac{\left(f_{d k}-f_{u k}\right)}{2}, \\
m_{0 k}=\sum_{k} \frac{\left(f_{d k}+f_{u k}\right)}{2} .
\end{gathered}
$$

Here $m_{u}$ and $m_{d}$ signify the ascending and descending magnetization, respectively, $h$ represents the field excitation, $h_{c k}$ is the coercivity of the $k$ th process. $a_{k}$ is the amplitude of the magnetization, $\alpha_{k}$ is a scaling factor, and $f_{o k}$ is the integration 
constant, while $h_{m}$ is the maximum field excitation, common to all. The index $k$ refers to the individual processes and $n$ is the number of total processes involved (for most substances $n=3)$.

The parameters are calculated by changing the model parameters until the best fit to the measured curve is achieved. When the iteration gives the best fit, the normalized and the equivalent physical values can be read from the two coordinate systems (normalized and measured), as shown in Figures 1, 2, and 3.

\section{References}

[1] F. Fiorillo, Measurement and Characterization of Magnetic Materials, Elsevier, Oxford, UK, 2004.

[2] D. Jiles, Introduction to Magnetism and Magnetic Materials, Chapman and Hall, New York, NY, USA, 1998.

[3] T. Nakata, N. Takahashi, K. Fujiwara, M. Nakano, Y. Ogura, and K. Matsubara, "An improved method for determining the DC magnetization curve using a ring specimen," IEEE Transactions on Magnetics, vol. 28, no. 5, pp. 2456-2458, 1992.

[4] F. Preisach, "Uber die magnetische Nachwirkung," Zeitschrift für Physik, vol. 94, pp. 227-302, 1935.

[5] E. C. Stoner and E. P. Wohlfarth, "A mechanism of magnetic hysteresis in heterogeneous alloys," IEEE Transactions on Magnetics, vol. 27, no. 4, pp. 3475-3518, 1991.

[6] J. Takacs, "The everett integral and its analytical approximation," in Advanced Magnetic Materials, L. Malkinsk, Ed., pp. 203-230, Intech, 2012.

[7] J. Takâcs, "A phenomenological mathematical model of hysteresis," Compel, vol. 20, no. 4, pp. 1002-1014, 2001.

[8] I. Meszaros, Private communication on $m_{0}$.

[9] H. Hauser, Y. Melikhov, and D. C. Jiles, "Examination of the equivalence of ferromagnetic hysteresis models describing the dependence of magnetization on magnetic field and stress," IEEE Transactions on Magnetics, vol. 45, no. 4, pp. 1940-1949, 2009.

[10] G. Bertotti, Hysteresis in Magnetism, Academic Press, San Diego, Calif, USA, 1998.

[11] D. C. Jiles and D. L. Atherton, "Theory of ferromagnetic hysteresis," Journal of Magnetism and Magnetic Materials, vol. 61, no. 1-2, pp. 48-60, 1986.

[12] J. A. Brug and W. P. Wolf, "Demagnetizing fields in magnetic measurements. I. Thin discs," Journal of Applied Physics, vol. 57, no. 10, pp. 4685-4694, 1985.

[13] A. Benabou, J. V. Leite, S. Clénet, C. Simão, and N. Sadowski, "Minor loops modelling with a modified Jiles-Atherton model and comparison with the Preisach model," JMMM, vol. 320, no. 20, pp. e1034-e1038, 2008.

[14] R. G. Harrison, "Physical theory of ferromagnetic first-order return curves," IEEE Transactions on Magnetics, vol. 45, no. 4, pp. 1922-1939, 2009.

[15] L. K. Varga, Gy. Kovacs, and J. Takacs, "Anhysteretic and biased first magnetization curves for Finemet-type toroidal samples," JMMM, vol. 320, no. 3-4, pp. L.26-L.29, 2008.

[16] J. Takacs, "Barkhausen instability and its implication in $\mathrm{T}(\mathrm{x})$ modelling of hysteresis," Compel, vol. 24, no. 4, pp. 1180-1190, 2005.

[17] J. Takacs, Mathematics of Hysteretic Phenomena, Wiley, Berlin, Germany, 2003. 

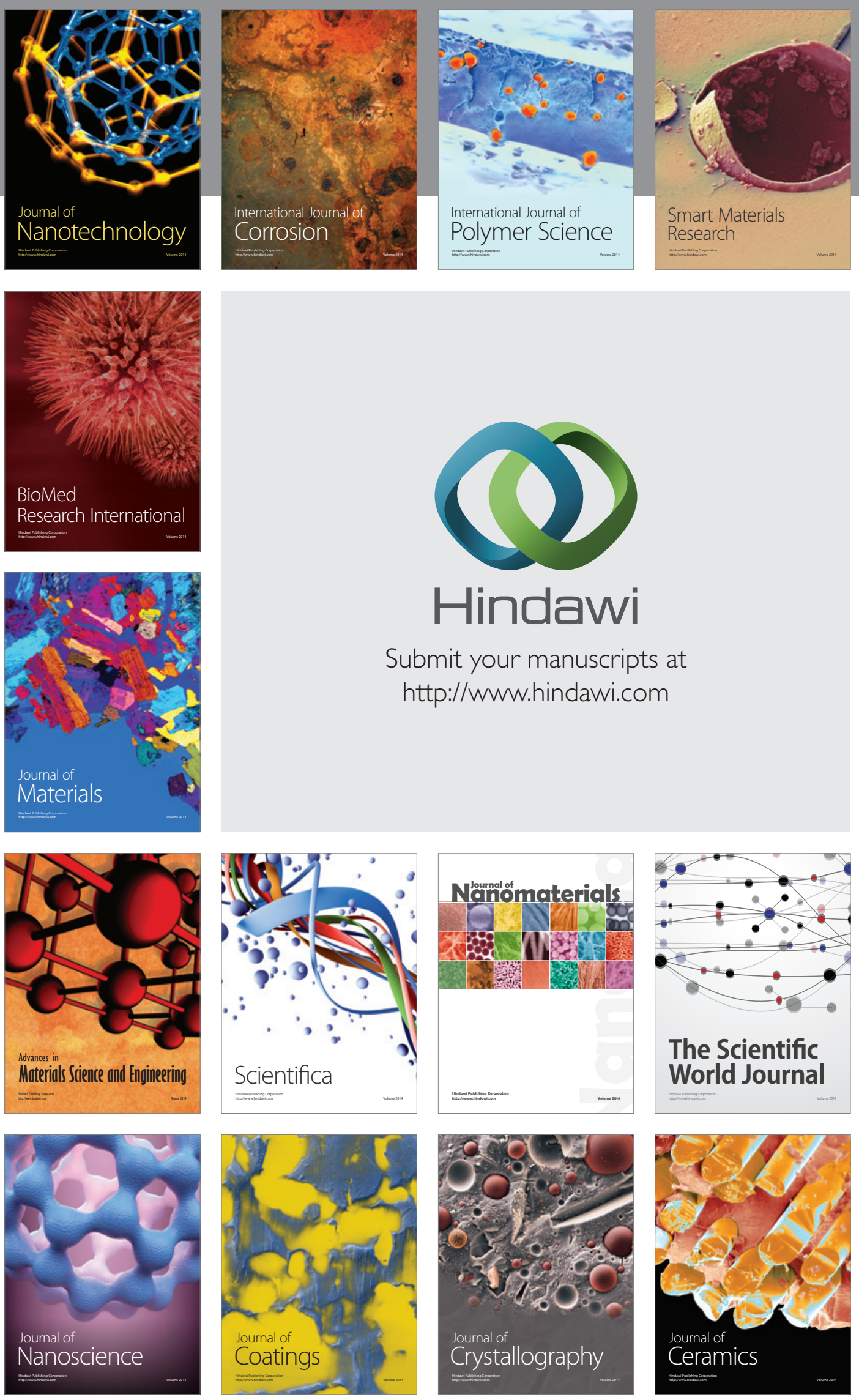

The Scientific World Journal

Submit your manuscripts at

http://www.hindawi.com

\section{World Journal}

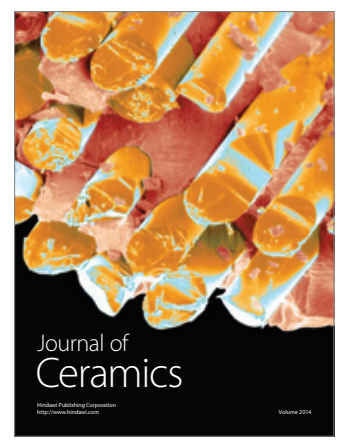

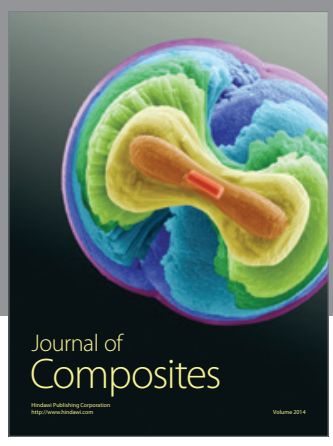
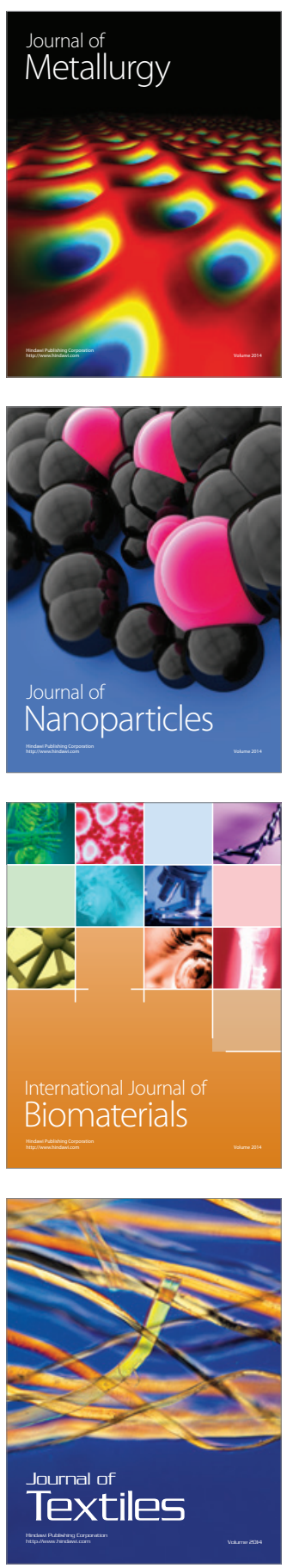